\title{
STABILIZATION OF VARIABLE BIT RATE VIDEO STREAMS USING LINEAR LYAPUNOV FUNCTIONS ON WiRELESS MOBILE NETWORK
}

\author{
Dr.V.Saravanan \\ PG Department of Information Technology, Hindusthan College of Arts and Science, \\ Coimbatore, India
}

\begin{abstract}
Streaming videos over wireless networks suffers from low video quality due to network ability limitations. The quality of the channel and the characteristics of source play the major role in transmitting video stream over mobile environments. On failure of wireless video transmission, retransmission method was employed to improve the reliability of wireless link. However, retransmission of video leads to significant impact on energy consumption and bounded average waiting time is also increased. The longer waiting time on retransmission results in buffer starvation. Therefore, it is desirable to reduce the variable bit rate of transmitted video signal and increase the stability level when buffer starvation is occurs. In order to overcome such limitation, a technique named Response based Stabilization Analysis (RSA) using Distributed Optimality Bit Rate Allocation (RSA-DOBRA) is proposed in this paper. Initially, video stream is segregated into frames of different classes (i.e., size). Each frame is transmitted based on the variable bit rate response using Optimal Quantization process. Secondly, Linear Lyapunov Functions is employed with RSA to prove the stability of different bit rates on wireless video streaming. The application of Linear Lyapunov Function maintains the stability level of bit rate on different class of frame transmission on wireless link. Finally, Distributed Optimality Bit Rate Allocation uses the time slicing procedure to reduce the bounded average waiting time. RSA performs the time slicing based on multiplexed wireless video transmission on variable bit rate to avoid buffer starvation. RSA at the final stage reduces the energy consumption by improving the reliability of wireless link. Experiment is conducted on factors such as buffered starvation rate, waiting time on video frame transmission, and energy consumption rate.
\end{abstract}

\section{KEYWORDS}

Optimality Bit Rate Allocation, Stabilization Analysis, Linear Lyapunov Functions, Variable Bit Rate, Time Slicing, Multiplexed Wireless Video Transmission

\section{INTRODUCTION}

Rapid development in wireless mobile networks is intensifying the desire that services conventionally available in wire-line networks, including audio, video, are also made available to wireless mobile users. However, several important issues, such as variable bit rate, are unique to wireless mobile networks and justify notable attention. Mobile Multimedia Streaming Techniques (MMST) [1], impact of energy consumption was analyzed using power saving mechanisms. Traffic aware QoS (T-QoS) [2] was designed to address the aspects related to the quality of service measures which improved the throughput being measured at traffic delay. Burst Scheduling Procedure (BSP) [3] introduced the concept of base station that constructed a schedule for transmission for different TV channels. However, in all the above methods, the DOI : $10.5121 /$ ijait.2016.6301 
retransmission of video resulted in significant impact on energy consumption and bounded average waiting time adversely affecting the buffer starvation.

With the advance swift in the mobile video stream traffic and heavier design and deployment of Base Stations has resulted in the efficient design of networks in an imperative manner. Prediction Green Streaming (PGS) [4] optimization method was designed to yield significant amount of energy savings using Mixed Integer Linear Program (MILP). Based on the estimation of capacities, a Buffer-based approach [5] was designed to improve the delivery rate of video. Two buffering architectures were designed in [6] to address the issues related to Quality of Service. The method not only improved the throughput but also significantly reduced the contention level. However heterogeneous data remained unaddressed.

With the inception of mobile hand-held devices the profusion of advances established in computers and the design of network systems with high speed have resulted to surge of video streaming. A Network Bandwidth Switcher (NBS) [7] introduced a feedback fuzzy controller to address different stream videos ranging from low to high quality video streams. However, the quality of content over Internet remained unaddressed. Many recent research works have focused on the vital role played by quality-of-experience $(\mathrm{QoE})$ in Internet video frames.

For efficient delivery of videos a novel mechanism was designed in [8] to measure QoE and energy consumption were analyzed which resulted in optimal playback buffer configurations. A live streaming system called LAYSTREAM [9] was designed to address issues related to management of membership, construction of topology and effective transmission of video packets. Adaptive Streaming (AS) [10] algorithms were designed for delivering of good quality video files through Internet using in network resource management framework.

In this work, focus is made on Response based Stabilization Analysis (RSA) using Distributed Optimality Bit Rate Allocation (RSA) to reduce variable bit rate of transmitted video signal and increase stability level when buffer starvation occurs. The contributions of RSA method include the following:

1. To reduce the variable bit rate of transmitted video signal and increase the stability level when buffer starvation occurs with the aid of Response based Stabilization Analysis (RSA) using Distributed Optimality Bit Rate Allocation.

2. To maintain and prove the stability level of different bit rates on wireless video streaming using Linear Lyapunov Functions with RSA.

3. To reduce the bounded average waiting time using Distributed Optimality Bit Rate Allocation with the aid of time slicing procedure.

4. To avoid buffer starvation by performing time slicing based on multiplexed wireless video transmission on variable bit rate.

5. To reduce the energy consumption by improving the reliability of wireless link.

The structure of this paper is as follows. In Section 1, the basic problems related to video streaming in mobile networks are described. Section 2 demonstrates the related work. In Section 3, an overall framework of Response based Stabilization Analysis (RSA) using Distributed Optimality Bit Rate Allocation (RSA) method is presented. Section 4 describes the experimental setup. Section 5 provides an outline implementation results with parametric factors and present the resultant graph forms with the help of table and graph values. Finally, Section 6 concludes the work. 
International Journal of Advanced Information Technology (IJAIT) Vol. 6, No.2/3, June 2016

\section{RELATED WORKS}

As the growth of wireless mobile networks outperforms that of mobile networks, adaptive video streaming has become the need of the hour to match changing network conditions. An in network resource management framework [11] was designed to obtain a balance between resource allocation and quality of experience. But with varying scheduling the framework did not provide optimality in resource allocation. An adaptive video streaming [12] to address the issues related to scheduling was introduced that not only addressed with variable bit rate but also for differing topology. However based on the client's need scheduling of video streams became complicated. A client rate adaptation algorithm was designed in [13] for HTTP-based Adaptive Streaming (HAS). A Network Utilization Maximization (NUM) [14] formulation was introduced to address the issues related to pre-buffering and re-buffering.

The most important technical issues to be addressed over Internet are the requirement of addressing the recent updation in network conditions. With the changing network conditions, there arises higher packet loss and making the delay in packets to fluctuate a lot. The problem of coding rate control was addressed in [15] using leaky bucket model. An approach called as Content-Aware Distortion Fair (CADF) [16] was designed to minimize the computation and communication overhead while sending video frames. However, quality of experience remained unaddressed. Model based predictive control [17] was structured with the main objective of delivering good quality of video over Internet. But bandwidth stability remained an open issue to be addressed.

One of the most important factors to be considered during HTTP streaming is the determination of availability of resource the demand for the same. In [18], bit rate estimation with bandwidth stability was addressed for the estimation of throughput. Fair-share bandwidth was introduced in [19] for minimizing the instability in the selection of video bit rate using an algorithm called PANDA. However, the quality of transmission gets affected with variable video bit rate. To address this issue, Adaptive Opportunistic Network Coding (AONC) [20] was designed to not only improving the transmission quality of video being sent on Internet but also the bandwidth and energy resources utilized.

A dynamic routing algorithm called as Mobility Assisted Dynamic Routing (MADR) was presented in [21] for the mobile wireless sensor networking system in which both the base station and the sensor nodes are in moving condition. MADR algorithm was designed to improve the throughput and to reduce the energy consumption in mobile wireless sensor networking system. Cycle-Based Minimum-Cost Domain-Disjoint Paths Algorithm was developed in [22] where spanning tree and balanced tree were employed to reduce bandwidth overhead, delivery delay and to increase throughput and packet delivery ratio. Matrix Based Bit Jumbling Technique (MBBJT) was designed in [23] that take the plain text (i.e. the input file) as a binary bit stream with finite number bits and ensure high security in message transmission of any form and is appropriate for any sort of file transfer 


\section{VAriable Bit Rate Video Streams Stabilization ON Wireless MOBILE NETWORK}

The primary goal of wireless video transmission in our proposed work is to optimize the video quality with effective throughput level on the channel. The performance of source channel in wireless network is monitored using Optimal Quantization Procedure. The Response based Stabilization Analysis (RSA) using Distributed Optimality Bit Rate Allocation Algorithm contains both the source point and destination point while transmitting the video packets. Video streaming at the source point is linked to wireless router and variable bit rates are administered. The variable bit rate at the source point produce better quality of video rate with limited bounded waiting time using RSA method. Video Streaming on wireless mobile network using RSA is depicted in Figure 1.

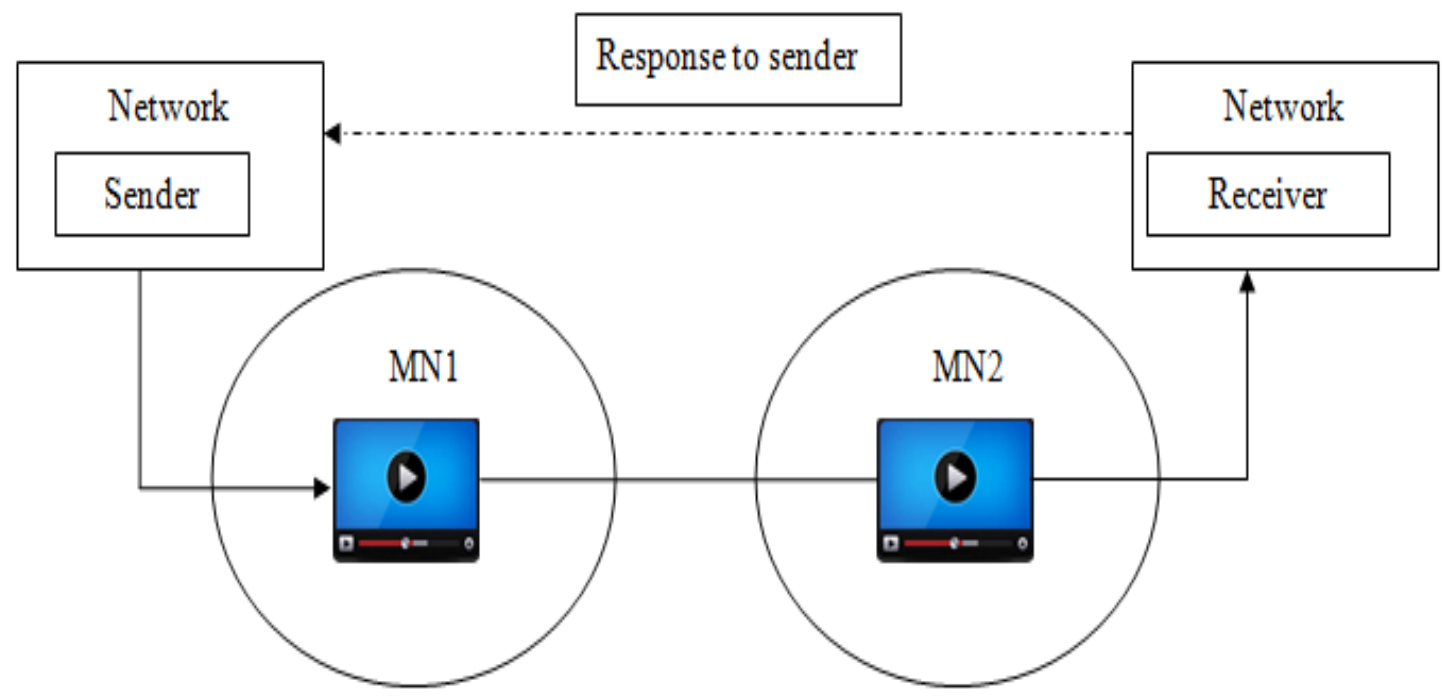

\section{MN-Mobile Network}

Figure1: Video Streaming on the Wireless Mobile Network

Figure 1 depicts the video streaming on wireless mobile network. The wireless mobile network based video transmission stream path is described in Figure 1. The stream involves packet delivery of frames sequentially on wireless link. RSA helps to transmit the packet with high quality rate from sending end to the destination end. In return, the response is produced from the destination to source point that indicates the acknowledgement of effective quality video being transmitted. The main aim of the RSA system is to design a wireless system for quality and stabilized bit rate video transmission. The architecture diagram of Response based Stabilization Analysis (RSA) is depicted in Figure 2. 


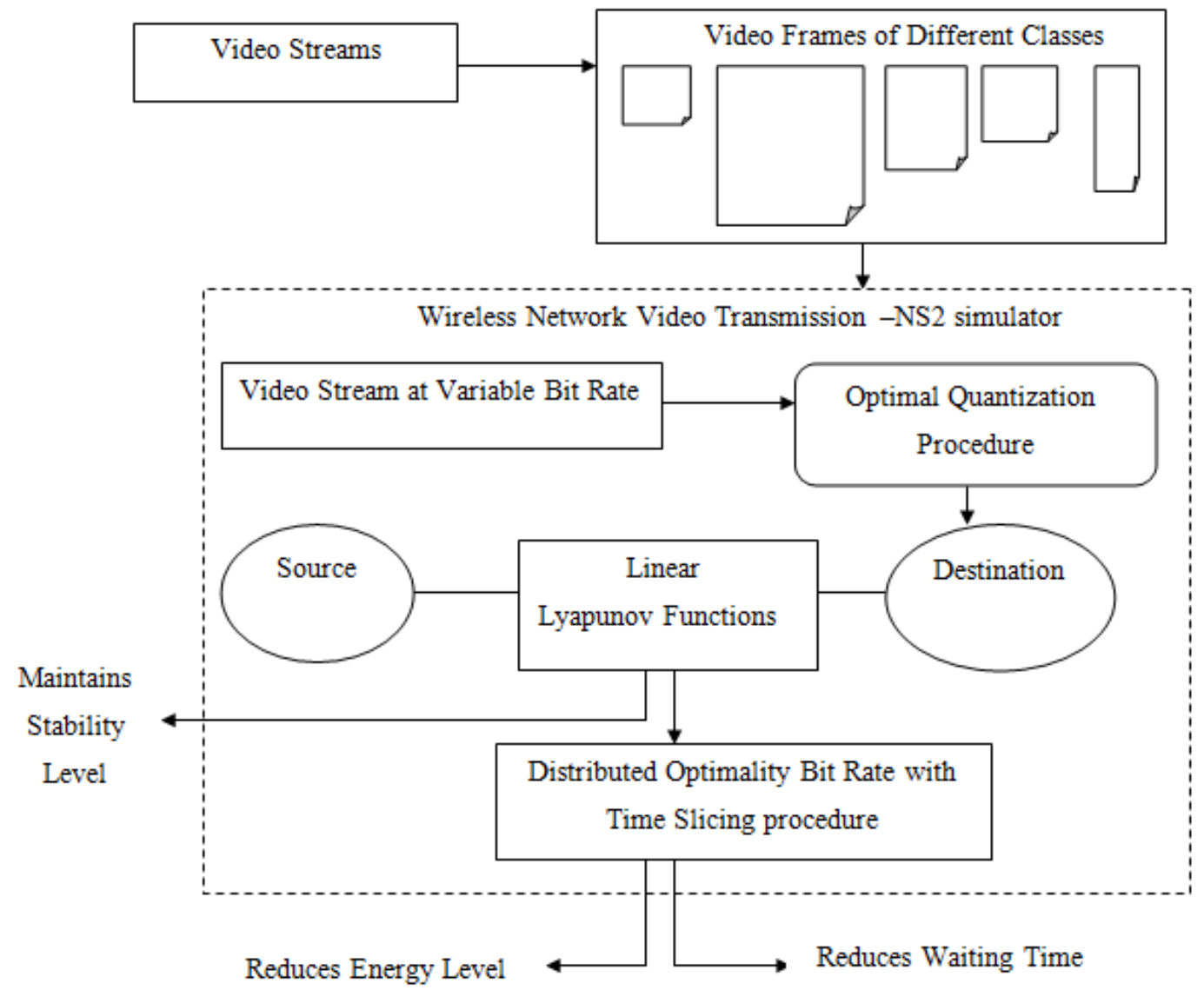

Figure 2 Architecture Diagram of Successful Video Transmission in Wireless Mobile Network Using Response based Stabilization Analysis

The transmission of video stream frames of different classes over wireless mobile network is illustrated in Figure 2 using Response Stabilization Analysis method. The wireless video streaming uses the NS2 simulator tool to collect all the segregated frames and perform high quality achievable video transmission. Video transmission through variable bit rate in wireless network produce better quality and an optimization quantization process is carried out to reduce the bounded waiting time.

Source node is the starting point in wireless video streaming that transmit the frames to the other end of the communication (i.e., destination end). The larger the wireless network structure the higher the video packets transmitted to different users (i.e., nodes). The quality of RSA is clearly monitored using the optimization Quantization process with Linear Lyapunov Functions. The function clearly stabilizes the bit rate on transmitting each frame from source to destination with minimal bounded waiting time. The stabilization is analyzed by the response function obtained from the receiver side. The RSA uses Distributed Optimality Bit Rate Allocation Algorithm that establishes the relative simplicity and improve the combined overall quality of the video with minimal energy level. 


\subsection{Optimal Quantization Process}

The initial design considerations of RSA-DOBRA include the segregation of video frames of different classes based on the variable bit rate using Optimal Quantization process. In this section, the design of Optimal Quantization process is discussed with the aid of mathematical formulation. The RSA method considers the wireless video streaming system to deliver the data frames between the mobile users via Access Point (AP). Using Optimal Quantization process, the transmitted video is encoded using scalable quantization technique. Scalable Quantization segregates the different size video streams into a set of countable size of the smaller set to improve the throughput rate. The throughput level $T$ using RSA method is the ratio of video

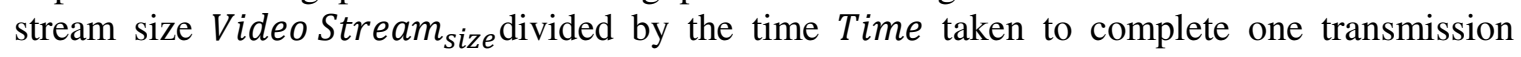
between source and destination.

$T=\frac{\text { Video }_{\text {Stream }} \text { size }}{\text { Time }}$

Let us consider a scenario that performs Scalar Quantization for RSA groups and rounds all the smaller set of video frames expressed as given below,

$$
S Q(f)=\operatorname{optimal}(S(F)) \cdot \Delta\left[\frac{f}{\Delta}+\frac{1}{2}\right]
$$

Scalar Quantization $S Q$ over ' $f$ ' frames from video stream is optimally quantized with signum quantized function using RSA method. The signum quantized function $(S(f))$ is a function used to round up the smaller set of video frame values consisting of ' $\Delta$ '. The Optimal Quantization process in RSA with variable bit rate monitors the channel state and also the buffer occupancy level. The bit rate stream value allotted to maintain the stability level is assigned as' $\Delta$ '. The property of RSA optimized quantization is that it has a countable set of stabilized bit rate value to improve the video quality rate on wireless mobile network.

The optimal quantization step with scalar factor is relatively simple that shows the rounding operation with lesser buffer starvation rate. The buffered starvation occurs when the transmission of the video frames fails to transmit to the destination through the intermediate points. The buffered starvation rate $B S R$ using RSA method is the summation of the frame combined $f_{1}+f_{2}+\cdots f_{n}$ based on the variable bit rate $V B R$ given as

$B S R=\operatorname{VBR}\left(f_{1}+f_{2}+\cdots f_{n}\right)$

In RSA method, retransmission of the video frames is carried out by achieving the minimal starvation rate frame structure through the quantization activities. The source point performs the forward quantization for developing the effective wireless video transmission communication channel of different bit rates. The stability of different bits rates can be improved by Linear Lyapunov Functions which is discussed in the next section. 


\subsection{Linear Lyapunov Functions}

Once the segregation of video frames is performed efficiently for different classes using optimal quantization process, Linear Lyapunov Functions is employed with RSA to prove the stability of different bit rates on wireless video streaming. With this, the application of Linear Lyapunov Function maintains the stability level of bit rate on different class of frame transmission on wireless link.

For effective stabilization of bit rate on wireless video transmission, scalar quantization is used with Linear Lyapunov functions. The concept behind the Linear Lyapunov functions is that it extracts the positive values to stabilize the bit rates with higher response acknowledgment. The response are clearly obtained from the destination side and Linear Lyapunov functions is formularized as,

$$
L L F=F(f) \geq 0
$$

From (4), the function $F(f)$ takes the positive values for maintaining the stability rate on variable bit rate video transmission. The stabilization of the bit rate with the locally positive definite derivative is defined as,

$$
L L F=F(f) \geq 0 \quad \forall(f \in N)
$$

The function denotes the video frames ' $f$ ' within the same stability of the channel bit rate whereas the ' $N$ ' denotes the neighborhood nodes, the frame (i.e., packets) transmitted in the wireless network with stabilized bit rate. The Linear Lyapunov functions on localized mobile network nodes are described as,

$$
L L F=F(f) \geq 0 \forall(f \in N[M N 1])
$$

The Linear Lyapunov Function $(L L F)$ on localized wireless video transmission network denotes the packet transfer for specified network zone structure. The frames transmitted from the source point are collected and transmitted to the neighboring ' $N$ ' nodes within the mobile network zone ' 1 '. In the similarly manner, the overall packet transfer within the localized wireless network structure is carried out through (6). The Linear Lyapunov functions on globalized mobile network nodes are described as,

$$
L L F=F(f) \geq 0 \forall(f \in N[M N 1, M N 2 \ldots . M N n])
$$

The globalized procedure in wireless video stream using Linear Lyapunov functions maintains the stability level of variable bit rate. The transfer of frames through the AP link to different network structure zone and finally reaches the destination point with optimal stabilized analysis bit rate. Once the stability of bit rates for different classes are achieved, the bounded average 
waiting time is reduced using the Distributed Optimality Bit Rate Allocations which is described in the next section.

\subsection{Distributed Optimality Bit Rate Allocation}

Finally, RSA uses Distributed Optimality Bit Rate Allocation with the aid of time slicing procedure to minimize the bounded average waiting time. Also RSA also buffer starvation by performing time slicing based on multiplexed wireless video transmission on variable bit rate. The Waiting Time on video transmission $W T_{\text {video transmission }}$ using RSA method represents the time taken to perform the frame distribution consisting of frames $f_{1}, f_{2}, \ldots, f_{n}$ as given below. It is measured in terms of milliseconds (ms).

$W T_{\text {video transmission }}=\operatorname{Time}\left(f_{1}+f_{2}+\ldots+f_{n}\right)$

In order to reduce the energy level and waiting time using RSA method, the distributed frames based on variable bit rate are allocated together on the receiving end. The allocation of bits includes the set of different frame classes in order to produce the optimality result. RSA method combines all the frames of the video stream and reduces the starvation with the aid of Distributed Optimality Bit Rate Allocation in wireless network. The Time Slicing procedure employed in RSA method is briefly described in section 3.3.1.

\subsubsection{Time Slicing Procedure}

The energy consumption and the bounded waiting time are reduced in the proposed work by applying time slicing procedure. At the same time, the RSA method also performs the time slicing procedure based on multiplexed wireless video transmission on variable bit rate to avoid the starvation on buffer space. The amount of energy consumed to transmit video of packet $\mathrm{n}$ of size $P(n)$ bits at a rate of $Q(n)$ bits/s using RSA method on buffer space $\mathrm{B}$ is given as follows

$E(n)=\sum B_{n, l},(Q(n))$

Where $B_{n, l}$ denotes the $\mathrm{n}$ packet of video transmission with a variable bit rate based on $l$ multiplexed blocks. Time Slicing is represented is Figure 3. 


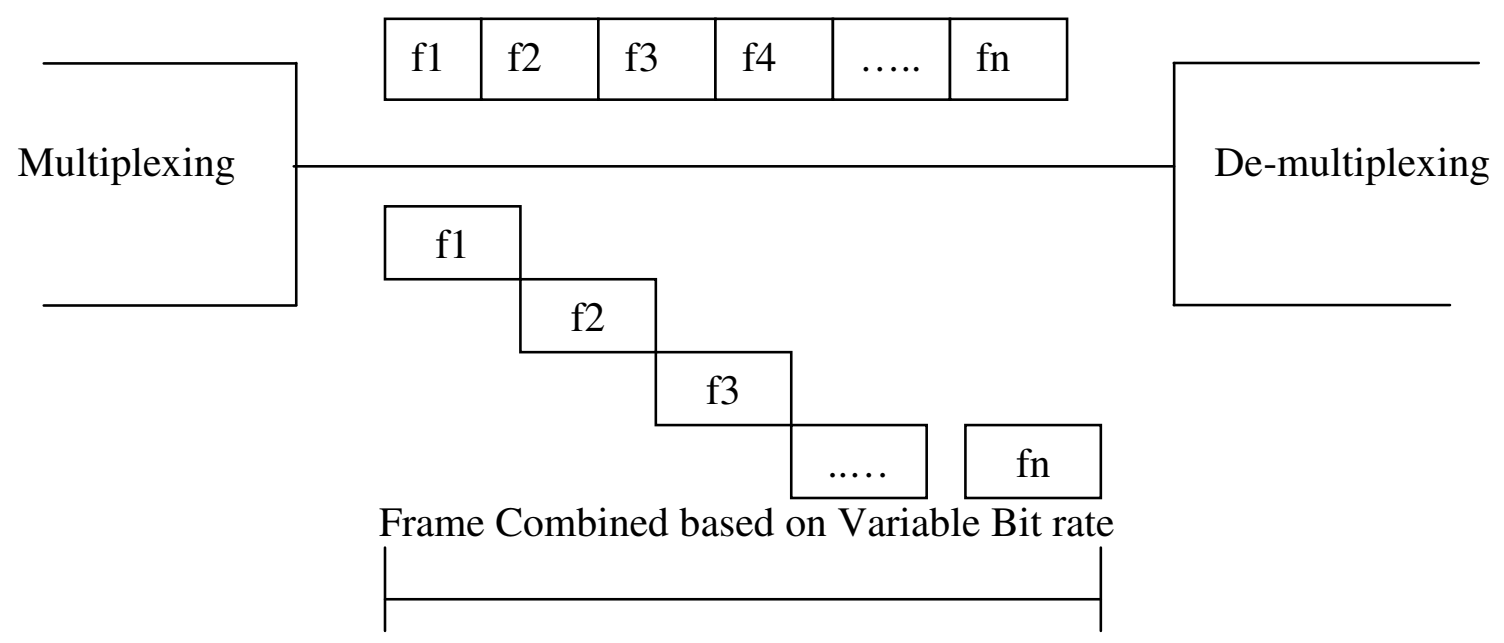

Figure3.Time Slicing Procedure

With the help of Time Slicing procedure, the segregated frames of the video stream are combined with minimal bounded waiting time. The combining of the frames reduces the energy level through multiplexing.

\subsubsection{Algorithm Procedure}

Distributed Optimality Bit Rate Allocation (DOBRA) solves the problem of the bounded waiting time on allocating the bits of different classes. RSA method aims at stabilizing the target bit rate by optimally allocating bits of various classes. The algorithmic procedure is described as follows,

Input: Wireless Video Streaming with Variable Bit rate on Source ' $S$ ' and Destination ' $D$ '

Output: Stabilized Analysis of the rate on different classes

Step 1: Optimal Quantization Process with Scalar Quantization

Step 2: Distribute variable bit rate frames using the Linear Lyapunov functions

Step 2.1: Observe stability of the channel bit rate with ' $N$ ' neighboring nodes

Step 2.2: Apply Linear Lyapunov functions on localized mobile network nodes

Step 2.3: Compute Linear Lyapunov functions on globalized mobile network nodes

Step 3: Distributed bits are allocated based on the time slicing on wireless network

Step 4: Time slicing reduces waiting time and energy level which initially start with zero bits allocated for all classes in RSA 
The distributed optimality bit is allocated to stabilize the variable bit rate using scalar quantization. The rounding up of smaller video frame values improves the quality rate on the receiving side of wireless network. The Linear Lyapunov functions adopted in RSA method improves both the localized and globalized video transferring with stable bit rate. Finally the distributed bit rate is allocated with optimal result of bounded waiting time and energy level.

\section{EXPERIMENTAL EVALUATION}

Response based Stabilization Analysis (RSA) using Distributed Optimality Bit Rate Allocation Algorithm is briefly explained using NS2 simulator. The simulation is carried out over the field area of size $1000 \mathrm{~m} \times 1000 \mathrm{~m}$. For the purpose of experimentation, NS2 simulation takes 50 nodes that are used for video transmission between source and destination with high quality rate. The video frames (i.e.,) packets are continuously transmitted in the wireless network on variable bit (i.e., speed) rate. The network uses DSR routing protocol to perform experiment on randomly moving frames to the destination side. The mobile nodes move at the random speed of $30 \mathrm{~m} / \mathrm{s}$ with an average pause of $0.01 \mathrm{~s}$.

Random Way Point (RWM) model is chosen to easily shift the video streams at a randomly chosen location. The randomly selected location with a different velocity offers predefined speed whereas the random progression is variable during the simulation period. RSA using Distributed Optimality Bit Rate Allocation Algorithm is compared against the existing Mobile Multimedia Streaming Techniques (MMST) [1], Traffic-aware QoS (T-QoS) [2] and Burst Scheduling Procedure (BSP) [3] in mobile TV networks and Mobility Assisted Dynamic Routing (MADR) algorithm [21]. Experiment is conducted on factors such as transmission rate, buffered starvation rate, waiting time on video frame transmission, throughput level.

\section{RESULTS AND DISCUSSIONS ON RSA}

To assess the performance of RSA method and comparison is made with existing methods namely, Mobile Multimedia Streaming Techniques (MMST) [1], Traffic-aware QoS (T-QoS) [2] and Burst Scheduling Procedure (BSP) [3] and Mobility Assisted Dynamic Routing (MADR) algorithm [21,] all five methods were implemented using the NS2 software. The underlying ad hoc routing protocol chosen was DSDV, which is suitable for DOBRA.

The results on RSA are investigated with the small stage information which is obtained from experimental work. A video frame comprises of many video stream size with differing video frame rate with CPU core equivalent to 500, 1000 and 1500 Microprocessor. 4GB RAM is used for the experimental work and $1 \mathrm{~TB}$ of storage of single domain information. The proposed RSA is compared against the existing Mobile Multimedia Streaming Techniques (MMST) [1], Traffic-aware QoS (T-QoS) [2], Burst Scheduling Procedure (BSP) [3], Mobility Assisted Dynamic Routing (MADR) algorithm [21]. The RSA method conducts experimental work on factors such as buffered starvation rate, waiting time on video frame transmission, throughput level. 
International Journal of Advanced Information Technology (IJAIT) Vol. 6, No.2/3, June 2016

Table 3.2.Tabulation for Buffered Starvation Rate with varied sizes of video streams

\begin{tabular}{|c|c|c|c|c|c|}
\hline \multirow{2}{*}{$\begin{array}{c}\text { Video } \\
\text { Stream } \\
\text { Size } \\
(\mathrm{Kbps})\end{array}$} & \multicolumn{5}{|c|}{ Buffered Starvation Rate (\%) } \\
\hline & $\begin{array}{l}\text { Proposed } \\
\text { RSA }\end{array}$ & $\begin{array}{l}\text { Existing } \\
\text { MMST }\end{array}$ & $\begin{array}{c}\text { Existing T- } \\
\text { QoS }\end{array}$ & $\begin{array}{l}\text { Existing } \\
\text { MADR }\end{array}$ & $\begin{array}{c}\text { Existing } \\
\text { BSP }\end{array}$ \\
\hline 30 & 32.35 & 37.45 & 40.47 & 42.29 & 45.47 \\
\hline 60 & 35.75 & 40.85 & 43.87 & 45.36 & 48.87 \\
\hline 90 & 38.35 & 43.45 & 46.47 & 48.21 & 51.47 \\
\hline 120 & 41.25 & 46.35 & 48.36 & 50.54 & 53.36 \\
\hline 150 & 42.58 & 47.68 & 50.70 & 53.65 & 56.70 \\
\hline 180 & 40.45 & 45.55 & 48.57 & 51.67 & 53.57 \\
\hline 210 & 43.25 & 48.35 & 51.39 & 54.87 & 61.39 \\
\hline
\end{tabular}

Table 1 shows the buffered starvation rate produced for the different video stream. In addition, the buffered starvation rate produced for different video stream sizes is compared with four other methods, MMST [1], T-QoS [2], BSP [3], and MADR [21] respectively. From the values tabulated we can see that we have seven different instances considered for conducting the experiments using NS2 simulator.

To estimate the buffered starvation rate per nodes (i.e., per packet), the frames are combined and the summation of the frames are performed based on the variable bit rate (3). The main packet types involved in measuring the average buffered starvation rate are shown in Table 1 . The bandwidth and the transmission range were assumed to be $3 \mathrm{Mbps}$ and $500 \mathrm{~m}$, respectively, while the field area was selected to be of size set up to $1000 \mathrm{~m} * 1000 \mathrm{~m}$. The nodes were randomly distributed over the field area using random way point model. The minimum and maximum speeds of the nodes (i.e., packets) are set to be 10 and $30 \mathrm{~m} / \mathrm{s}$, respectively, and the average pause time to 0.01 seconds. 
International Journal of Advanced Information Technology (IJAIT) Vol. 6, No.2/3, June 2016

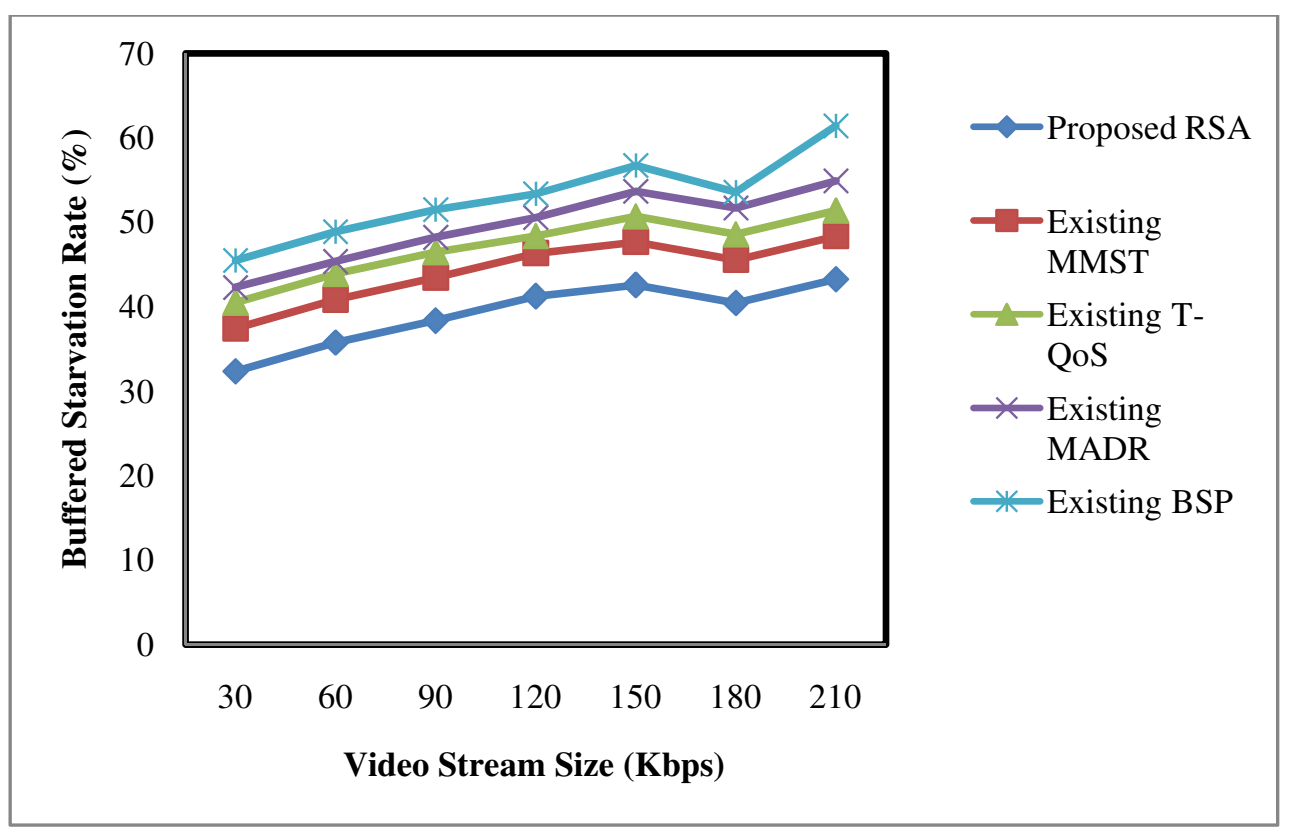

Figure 4.Impact of Buffered Starvation Rate on video differing video streams

Figure 4 illustrates the impact of changes in the buffered starvation rate on different video stream sizes. As illustrated in this graph, this buffered starvation rate increases linearly with the increase of video stream size. The increase in buffered starvation rate is justified since more source nodes with different video stream sizes will be sending their packets, and these requests must be forwarded to destination nodes, which will increase the buffered starvation rate on each node and, consequently, on the entire network.

The reason behind the improvement of buffered starvation rate is observed to be better by performing time slicing based on multiplexed wireless video transmission on variable bit rate. The lesser buffer starvation rate is achieved through the quantization activities with scalar factor that shows rounding operation with lesser buffer starvation rate by $13 \%$ and $21 \%$ better than MMST [1] and T-QoS [2] respectively. With the application of Scalable Quantization that uses Optimal Quantization process to segregate different size video streams into countable size of smaller set results in the minimized buffered starvation rate by $35 \%$ as compared to BSP [3] and $27 \%$ as compared to MADR [21] respectively . 
International Journal of Advanced Information Technology (IJAIT) Vol. 6, No.2/3, June 2016

Table 2.Tabulation for waiting time on Video Frame Transmission with video stream sizes measured in the range of $30 \mathrm{Kbps}$ and $210 \mathrm{Kbps}$

\begin{tabular}{|c|c|c|c|c|c|}
\hline \multirow{5}{*}{$\begin{array}{c}\text { Video } \\
\text { Stream } \\
\text { Size } \\
(\text { Kbps })\end{array}$} & $\begin{array}{c}\text { Waiting time on Video Frame Transmission (ms) } \\
\text { Proposed }\end{array}$ & $\begin{array}{c}\text { Existing } \\
\text { MMST }\end{array}$ & $\begin{array}{c}\text { Existing } \\
\text { T-QoS }\end{array}$ & $\begin{array}{c}\text { Existing } \\
\text { MADR }\end{array}$ & $\begin{array}{c}\text { Existing } \\
\text { BSP }\end{array}$ \\
\hline 30 & 25 & 30 & 31 & 34 & 36 \\
\hline 60 & 38 & 43 & 45 & 47 & 49 \\
\hline 90 & 31 & 36 & 37 & 39 & 42 \\
\hline 120 & 34 & 39 & 42 & 44 & 46 \\
\hline 150 & 32 & 37 & 39 & 41 & 44 \\
\hline 180 & 36 & 41 & 43 & 45 & 47 \\
\hline 210 & 41 & 46 & 48 & 50 & 53 \\
\hline
\end{tabular}

Results for the video frames of different video stream size (i.e.,) seven different packets are given in Table 2. The waiting time for each video stream size is observed and in a similar manner, comparison is made with other methods to obtain the waiting time. The waiting time is observed to be linearly increased till the video stream size is $120 \mathrm{Kbps}$. But for higher video stream sizes, the waiting time observed is not linear.

In order to observe the waiting time required for achieving 75 percent confidence level, s scenario with default parameters value of seven times was run. For each simulation run, the frame distribution rate and the time taken to obtain the frame distribution rate was changed. The waiting time on video frame transmission rate was computed starting from a video stream size with $f=30$ Kbps and $f=210 \mathrm{Kbps}$. As a result, the result of each scenario was computed with an average of seven simulation runs. 


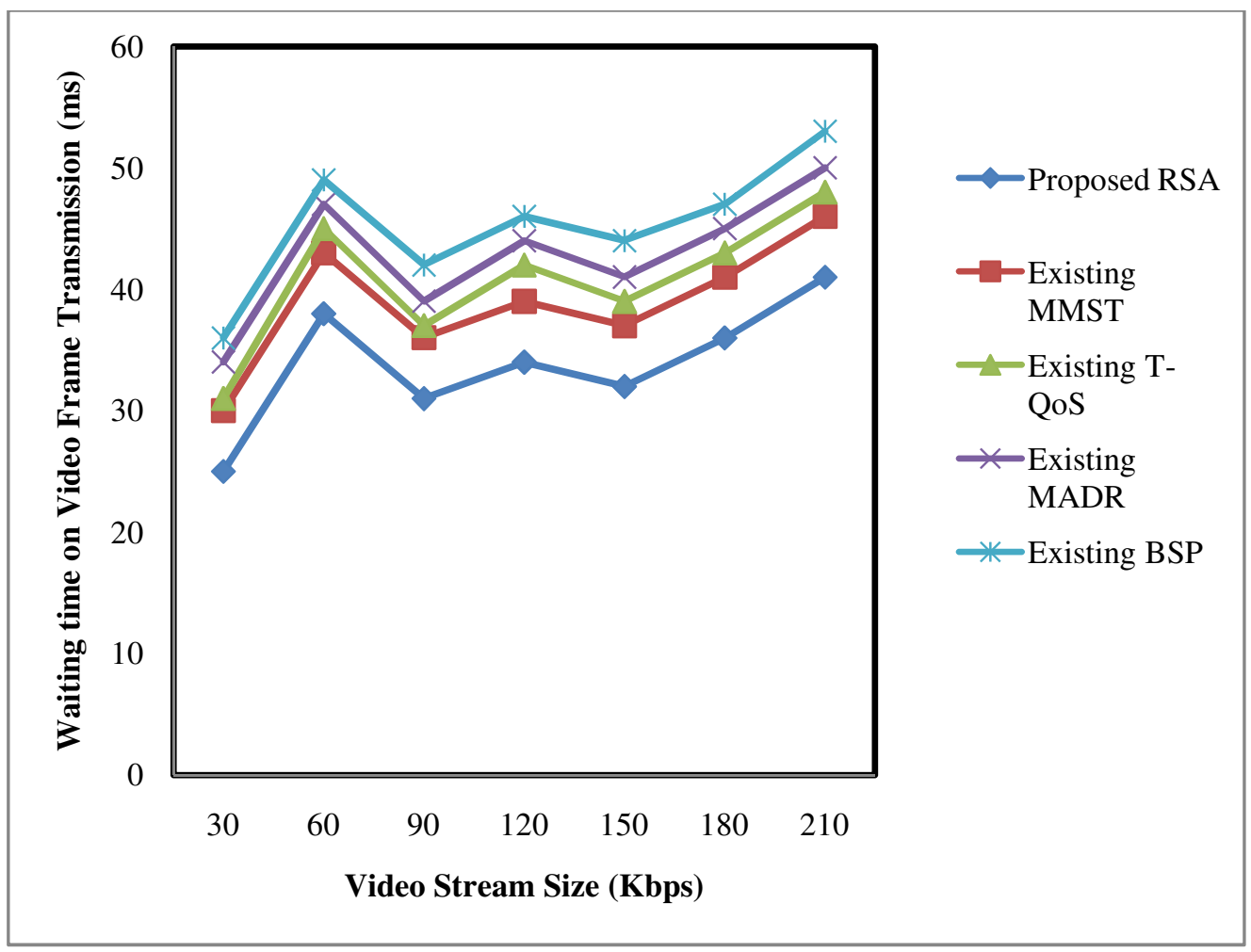

Figure 5 Impact of waiting time on Video Frame Transmission on video differing video streams

Figure 5 compares the average waiting time on video frame transmission of RSA method to that of MMST, T-QoS, BSP and MADR for the same scenarios discussed above. In all scenarios, the RSA method clearly outperforms all three systems. As illustrated in the graphs, the waiting time on video frame transmission follows a decreasing trend until packets start moving after $62 \mathrm{Kbps}$ from the start of the simulation. It can be observed that at increasing video stream size, the waiting time of RSA method and four other methods increases. Moreover, RSA method performs better than state-of-the-art works [1] [2] [3] [21] that show that it is consistent with the results. The waiting time on video frame transmission and energy level is reduced using Distributed Optimality Bit Rate Allocation with the aid of time slicing procedure. With the aid of Time Slicing procedure, the segregated frames of the video stream are combined based on variable bit rate that helps in minimizing the energy level consumption through multiplexing. This is because the frame distribution is performed based on variable bit rate that are allocated together at the receiving end to produce the optimality results. This result in the minimization of waiting time on video frame transmission by $15 \%, 21 \%, 34 \%$ and $27 \%$ when compared to MMST [1], T-QoS [2], BSP [3], and MADR [21] respectively. 
International Journal of Advanced Information Technology (IJAIT) Vol. 6, No.2/3, June 2016

Table 3.Tabulation for Throughput Level with respect to video frame rate

\begin{tabular}{|c|c|c|c|c|c|}
\hline \multirow{2}{*}{$\begin{array}{c}\text { Video } \\
\text { Frame } \\
\text { Rate } \\
\text { (fps) }\end{array}$} & \multicolumn{5}{|c|}{ Throughput Level (Bytes/S) } \\
\cline { 2 - 6 } & RSA & $\begin{array}{c}\text { Existing } \\
\text { MMST }\end{array}$ & $\begin{array}{c}\text { Existing T- } \\
\text { QoS }\end{array}$ & $\begin{array}{c}\text { Existing } \\
\text { MADR }\end{array}$ & $\begin{array}{c}\text { Existing } \\
\text { BSP }\end{array}$ \\
\hline 12 & 0.50 & 0.45 & 0.43 & 0.41 & 0.39 \\
\hline 15 & 0.65 & 0.60 & 0.58 & 0.56 & 0.54 \\
\hline 18 & 0.71 & 0.66 & 0.64 & 0.62 & 0.6 \\
\hline 21 & 0.74 & 0.69 & 0.67 & 0.65 & 0.63 \\
\hline 24 & 0.72 & 0.67 & 0.65 & 0.63 & 0.61 \\
\hline 27 & 0.77 & 0.72 & 0.70 & 0.68 & 0.66 \\
\hline 30 & 0.80 & 0.75 & 0.73 & 0.71 & 0.69 \\
\hline
\end{tabular}

Seven combinations of video transmission between source and destination with varied video frame rate in terms of frames per second, which can be allowed selectively to each source node and send to the other end is tabulated in Table 3. There are trade-offs between throughput level and video frame rate, depending on the random speed of the mobile nodes. Table 2 shows the combination of modulations of throughput level of our method RSA and comparison made with four other methods. 
International Journal of Advanced Information Technology (IJAIT) Vol. 6, No.2/3, June 2016

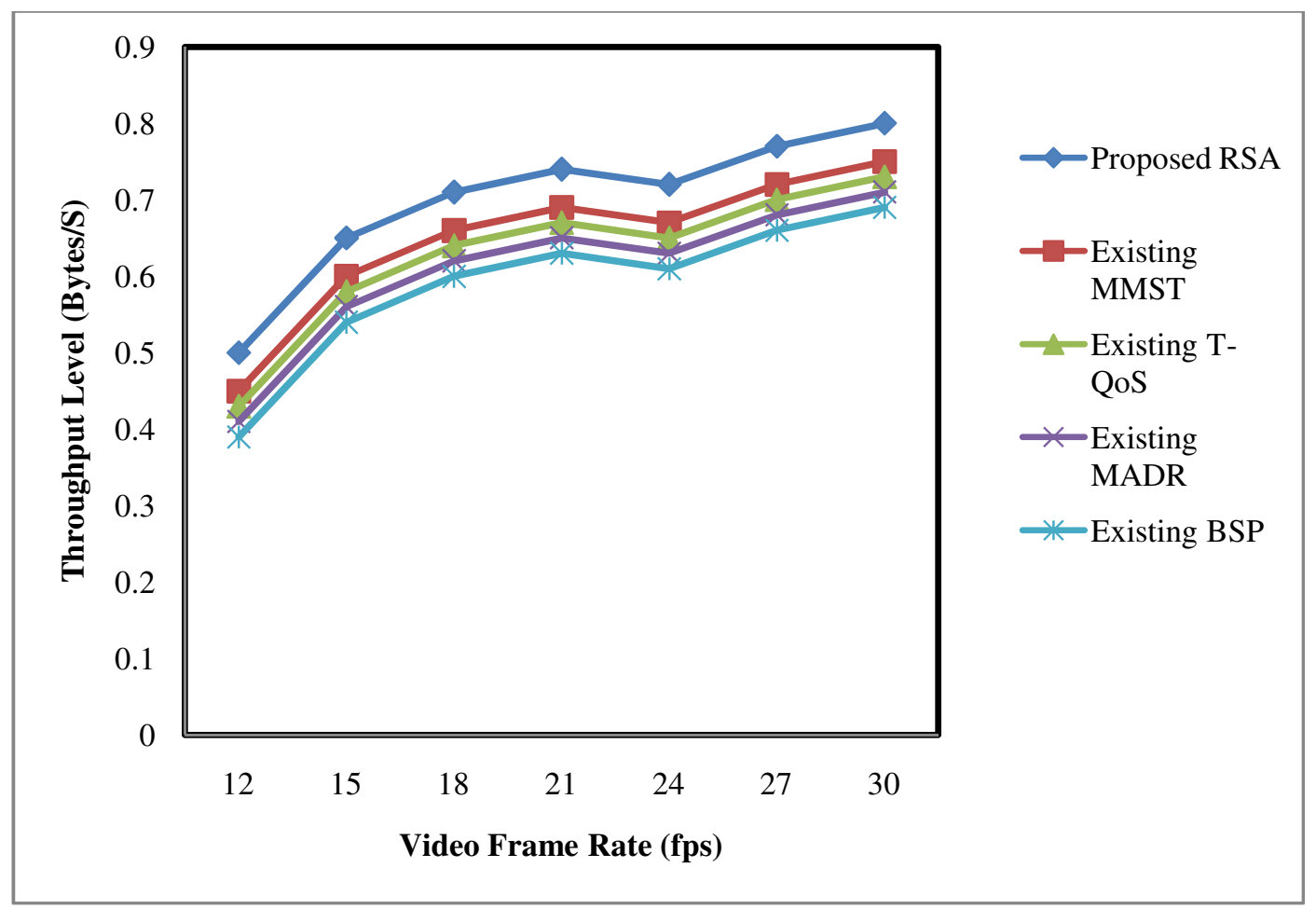

Figure 6.Impact of Throughput Level on video differing video frames

Figure 6 shows the behavior of the throughput level in response to varying video frame rate which was varied between $12 \mathrm{fps}$ and $42 \mathrm{fps}$. The average throughput level of the four methods increases with increasing video frame rate because more packets are sent between the sender and receiver nodes. The throughput level of RSA increases with increasing video frame rate up to 15 fps, where it starts increasing again at 24 fps. At low video frame rates, RSA method has high throughput level than MMST, T-QoS, BSP and MADR but the situation is reversed starting from a video frame rate of $30 \mathrm{fps}$, where RSA method starts to perform better. Finally, Figure 6 shows the throughput level of RSA method that is caused by the size of video streams at different time intervals. The throughput observed in the figure 6 finally, shows an observation that outperforms other three methods. This is because with the application of Linear Lyapunov Functions where the stability level of bit rate is maintained on different class frames on both localized and globalized mobile network nodes increasing the throughput level by $7 \%, 10 \%, 13 \%$ and $16 \%$ when compared to MMST [1], T-QoS [2], BSP [3], and MADR [21] respectively.

\section{CONCLUSION}

This paper presented a novel architecture for stabilizing of variable bit rate on wireless mobile network that segregates the video stream into frames and sending them based on the variable bit response to their destinations. A key feature of the system is its ability to increase the throughput level rapidly as more requests for video streams are transmitted to the destination. Optimal Quantization process and Linear Lyapunov Functions was employed for transmitting frames based on the variable bit rate and prove the stability of different bit rates on wireless video streaming. Finally, Distributed Optimality Bit Rate Allocation was deployed in an efficient 
manner to minimize the bounded average waiting time on wireless mobile network. Experimental evaluation and simulations analysis was conducted to analyze the robustness with respect to waiting time, buffer starvation rate and throughput level. Performances results reveal that the proposed RSA method provides higher level of throughput level and improved the buffer starvation rate and minimized the bounded waiting time on several simulation runs. Compared to the existing wireless mobile network methods, the proposed Response based Stabilization Analysis (RSA) method is comparatively better than compared to state-of-the-art works.

\section{REFERENCES}

[1] Mohammad Ashraful Hoquea, Matti Siekkinen, Jukka K. Nurminena, Mika Aalto, Sasu Tarkomac, "Mobile multimedia streaming techniques: QoE and energy saving perspective," Pervasive and Mobile Computing., Elsevier Journal., 2014

[2] Ravikumar Balakrishnan, and Berk Canber, "Traffic-Aware QoS Provisioning and Admission Control in OFDMA Hybrid Small Cells," IEEE TRANSACTIONS ON VEHICULAR TECHNOLOGY, VOL. 63, NO. 2, FEBRUARY 2014

[3] Cheng-Hsin Hsu, and Mohamed M. Hefeeda, "Broadcasting Video Streams Encoded With Arbitrary Bit Rates in Energy-Constrained Mobile TV Networks," IEEE/ACM TRANSACTIONS ON NETWORKING, VOL. 18, NO. 3, JUNE 2010

[4] Hatem Abou-zeid, Hossam S. Hassanein, and Stefan Valentin," Energy-Efficient Adaptive Video Transmission: Exploiting Rate Predictions in Wireless Networks", IEEE Transactions on Vehicular Technology, Volume 63, Issue 5, Pages 2013-2026, Jun 2014

[5] Te-Yuan Huang, Ramesh Johari, Nick McKeown, Matthew Trunnell, Mark Watson," A BufferBased Approach to Rate Adaptation: Evidence from a Large Video Streaming Service", Proceedings of the 2014 ACM conference on SIGCOMM, Pages 187 - 198, Aug 2014

[6] Ruonan Zhang, Lin Cai, Jianping Pan, Xuemin (Sherman) She," Resource management for video streaming in ad hoc networks", Ad Hoc Networks, Elsevier, Aug 2010

[7] Pengcheng Xiong, Jialie Shen, Qingyang Wang, Deepal Jayasinghe, Jack Li, Calton," NBS: a network-bandwidth-aware streaming version switcher for mobile streaming applications under fuzzy logic control, Proceedings of the 2012 IEEE First International Conference on Mobile Services, Pages 48 - 55, Feb 2012

[8] Mohammad Ashraful Hoque, Matti Siekkinena, Jukka K. Nurminena, Mika Aaltob, Sasu Tark," Mobile Multimedia Streaming Techniques: QoE and Energy Consumption Perspective", Prevasive and Mobile Computing, May 2014

[9] Miguel Matos, Valerio Schiavoniy, Etienne Rivierey, Pascal Felbery, Rui Oliveira," LAYSTREAM: composing standard gossip protocols for live video streaming", 14-th IEEE International Conference on Peer-to-Peer Computing, Pages 1 - 10, Sep 2014

[10] Xiaoqi Yin Vyas Sekar Bruno Sinopoli," Toward a Principled Framework to Design Dynamic Adaptive Streaming Algorithms over HTTP", ACM, Oct 2014

[11] Jiasi Chen, Sampath Rangarajan, Mung Chiang," A Scheduling Framework for Adaptive Video Delivery over Cellular Networks", ACM, Sep 2013

[12] D.Bethanabhotla, G. Caire and M. J. Neely," Adaptive Video Streaming for Wireless Networks with Multiple Users and Helpers", IEEE Transactions on Computers, Apr 2014

[13] Zhi Li, Ali C. Begen, Joshua Gahm," Streaming Video over HTTP with Consistent Quality", Proceedings of the 5th ACM Multimedia Systems Conference, Pages 248 - 258, Jan 2014

[14] D. Bethanabhotla, G. Caire and M. J. Neely," Adaptive Video Streaming in MU-MIMO Networks", In Proceedings of CoRR, Jan 2014

[15] Cheng Huang, Philip A. Chou, Anders Klemets," Optimal Coding Rate Control for Scalable Streaming Media", In Proceedings of International Packet Video Workshop, Dec 2004

[16] Ying Li, Zhu Li, Mung Chiang, and A. Robert Calderbank," Content-Aware Distortion-Fair Video Streaming in Congested Networks", IEEE TRANSACTIONS ON MULTIMEDIA, VOL. 11, NO. 6, OCTOBER 2009 
[17] Xiaoqi Yin Vyas Sekar Bruno Sinopoli," Toward a Principled Framework to Design Dynamic Adaptive Streaming Algorithms over HTTP", ACM, Oct 2014

[18] Truong Cong Thang, Hung T. Le, Hoc X. Nguyen, Anh T. Pham, Jung Won Kang, Yong Man Ro,” Adaptive Video Streaming over HTTP with Dynamic Resource Estimation", Journals of Communications and Networks, Pages 635 - 634, Jan 2014

[19] Zhi Li, Xiaoqing Zhu, Joshua Gahm, Rong Pan, Hao Hu, Ali C. Begen, and David Oran,” Probe and Adapt: Rate Adaptation for HTTP Video Streaming At Scale", IEEE JOURNAL ON SELECTED AREAS IN COMMUNICATIONS, VOL. 32, NO. 4, APRIL 2014

[20] Hang Shen, Guangwei Bai, Lu Zhao, and Zhenmin Tang," An Adaptive Opportunistic Network CodingMechanism in Wireless Multimedia Sensor Networks", Hindawi Publishing Corporation International Journal of Distributed Sensor Networks Volume 2012

[14] D. Bethanabhotla, G. Caire and M. J. Neely," Adaptive Video Streaming in MU-MIMO Networks", In Proceedings of CoRR, Jan 2014

[15] Cheng Huang, Philip A. Chou, Anders Klemets," Optimal Coding Rate Control for Scalable Streaming Media", In Proceedings of International Packet Video Workshop, Dec 2004

[16] Ying Li, Zhu Li, Mung Chiang, and A. Robert Calderbank," Content-Aware Distortion-Fair Video Streaming in Congested Networks", IEEE TRANSACTIONS ON MULTIMEDIA, VOL. 11, NO. 6, OCTOBER 2009

[17] Xiaoqi Yin Vyas Sekar Bruno Sinopoli," Toward a Principled Framework to Design Dynamic Adaptive Streaming Algorithms over HTTP”, ACM, Oct 2014

[18] Truong Cong Thang, Hung T. Le, Hoc X. Nguyen, Anh T. Pham, Jung Won Kang, Yong Man Ro,” Adaptive Video Streaming over HTTP with Dynamic Resource Estimation", Journals of Communications and Networks, Pages 635 - 634, Jan 2014

[19] Zhi Li, Xiaoqing Zhu, Joshua Gahm, Rong Pan, Hao Hu, Ali C. Begen, and David Oran,” Probe and Adapt: Rate Adaptation for HTTP Video Streaming At Scale", IEEE JOURNAL ON SELECTED AREAS IN COMMUNICATIONS, VOL. 32, NO. 4, APRIL 2014

[20] Hang Shen, Guangwei Bai, Lu Zhao, and Zhenmin Tang," An Adaptive Opportunistic Network CodingMechanism in WirelessMultimedia Sensor Networks", Hindawi Publishing Corporation International Journal of Distributed Sensor Networks Volume 2012

[21] S.R.Boselin Prabhu, V.S.Sakkthi Vigneesh, Arun Babu, Anand.T.C, Dr.S.Sophia, "Mobility Assisted Dynamic Routing For Mobile Wireless Sensor Networks", International Journal of Advanced Information Technology (IJAIT) Vol.3, No.1 February 2013

[22] S Deepakraj and Dr.R Thamilselvan, "Efficient Routing Mechanism Using Cycle Based Network and K-Hop Security in Adhoc Networks", International Journal of Advanced Information Technology (IJAIT) Vol. 4, No. 1, February 2014

[23] Manas Paul1 and Jyotsna Kumar Mandal, "A Universal Bit Level Block Encoding Technique Using Session Based Symmetric Key Cryptography to Enhance the Information Security", International Journal of Advanced Information Technology (IJAIT) Vol. 2, No.2, April 2012.

\section{Author}

Dr.V.Saravanan received M.Sc(CS)., from Bharathiar University in 1999, completed M.Phil., from Manonmaniam Sundaranar University in 2002. Received MCA., from Periyar University in 2011, and Received Ph.D., from Manonmaniam Sundaranar University in 2016. Currently working in Associate Professor \& Head in PG Dept. of Information Technology, Hindusthan College of arts and science, Coimbatore. His research area is Network security in mobile networking.

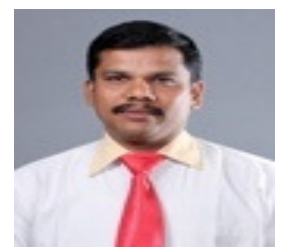

